\title{
Medo de quedas e atividades avançadas da vida diária em idosos
}

\section{Fear of falling and advanced activities of daily living in elderly}

\author{
Jéssica Faria Souto ${ }^{1}$, Pricila Cristina Correa Ribeiro ${ }^{2}$, Roberto Alves Lourenço ${ }^{3}$ \\ http://dx.doi.org/10.11606/issn.2238-6149.v29i3p215-222
}

Souto JF, Ribeiro PCC, Lourenço RA. Medo de quedas e atividades avançadas da vida diária em idosos. Rev Ter Ocup Univ Sao Paulo. 2018 set.-dez.;29(3):215-22.

RESUMO: Considerando que o medo de quedas é vivenciado inclusive por idosos que nunca caíram, podendo levar a limitações nas atividades de vida diária, o presente estudo investigou a associação entre medo de cair e Atividades Avançadas de Vida Diária (AAVD) em idosos comunitários. A amostra foi composta por 645 idosos (65 anos ou mais), clientes de uma operadora de saúde, de ambos os sexos, entrevistados pelo Estudo FIBRA-RJ. Para avaliar o medo de cair foi utilizado a Escala Internacional de Eficácia de Quedas - Brasil (FES-I-BR). As AAVD foram avaliadas com inventário baseado na literatura, sendo a manutenção de 7 ou mais AAVD, do total de 12, considerada como melhor funcionalidade. A prevalência de medo de queda foi $5 \%$ menor para idosos com pior funcionalidade para AAVD comparado àqueles com melhor funcionalidade. Esta associação entre o medo de cair e AAVD foi independente da fragilidade, idade, sexo, histórico de quedas, autopercepção de saúde, depressão, número de doenças crônicas e de medicação. Discute-se neste estudo se o desengajamento nas AAVD seria um mecanismo adaptativo na velhice e possíveis estratégias para redução do medo de quedas em idosos.

Descritores: Participação social; Idoso; Medo; Atividades cotidianas; Autoeficácia; Acidentes por quedas.
Souto JF, Ribeiro PCC, Lourenço RA. Fear of falling and advanced activities of daily living in elderly. Rev Ter Ocup Univ São Paulo. 2018 Sept-Dec;29(3):215-22.

ABSTRACT: The elderly population that never fell also experienced the fear of falls, which may lead to limitations in activities of daily living, this study investigated the association between fear of falling and Advanced Activities of Daily Living (AADL) in the elderly community. The sample consisted of 645 elderly ( 65 years or older), clients of a health care provider, of both sexes, interviewed by the FIBRA-RJ Study. The fear of falling was evaluated by the International Falls Efficiency Scale - Brazil (FES-I-BR). The AADLs were evaluated with literature-based inventory, with the maintenance of 7 or more AADLs, out of a total of 12 , considered as better functionality. The prevalence of fear of falling was $5 \%$ lower for the elderly with worse functionality for AADL compared to those with better functionality. This association between fear of falling and AADL was independent of fragility, age, sex, history of falls, self-perception of health, depression, number of chronic diseases and medication. This study discusses whether disengagement in AADL would be an adaptive mechanism in old age and possible strategies for reducing the fear of falls in the elderly.

Keywords: Social participation; Aged; Fear; Activities of daily living; Accidental falls.

$11^{\circ}$ Congresso Brasileiro de Psicologia Hospitalar, Gramado, RS, 2017. IX Congresso de Geriatria e Gerontologia de Minas Gerais. Belo Horizonte, MG, 2017. IX Seminário de Pesquisa em Gerontologia e Geriatria, Campinas, SP, 2016. VIII Congresso de Geriatria e Gerontologia de Minas Gerais, Ouro Preto, MG, 2015. $9^{\circ}$ Congresso Paulista de Geriatria e Gerontologia, São Paulo, SP, 2015.

1. Universidade Federal de Minas Gerais. ORCID: https://orcid.org/0000-0002-7085-4349. E-mail: jefsouto@gmail.com.

2. Universidade Federal de Minas Gerais. ORCID: https://orcid.org/0000-0001-9431-2707. E-mail: pricilaribeiro@ufmg.br.

3. Universidade do Estado do Rio de Janeiro. ORCID: https://orcid.org/0000-0003-0838-1285. E-mail: roberto.lourenco@globo.com. Endereço para correspondência: Jéssica Faria Couto. Rua Manoel Silverio Veloso, 25. Bairro California. Bocaiuva, MG. CEP: 39390000 . 


\section{INTRODUÇÃO}

$\mathrm{O}$ medo da queda em idosos pode levar a depressão, perda da confiança, diminuição da qualidade de vida, limitações na vida diária, falta de condicionamento físico, alterações do equilíbrio e da marcha, redução ou restrição de atividades funcionais e de contatos sociais ${ }^{1-3}$. Embora seja um fenômeno mais frequente entre idosos com histórico de quedas, o medo de quedas é relatado, inclusive, pelo público mais velho que nunca caiu ${ }^{4}$.

O julgamento do medo de quedas tem sido realizado, com maior frequência, a partir da teoria cognitiva social de Bandura, a partir da qual a autoeficácia relacionada às quedas é avaliada com a informação do quão confiante a pessoa é de que pode realizar suas atividades diárias sem cair ${ }^{5}$. De acordo com Tinetti et al. ${ }^{6}$, o medo de cair expressa baixa autoeficácia relacionada às quedas, isto é, falta de confiança na própria capacidade de evitar que as quedas aconteçam. Assim, a autoeficácia, compreendida como componente cognitivo do medo, é também um critério a ser incluído para avaliação da funcionalidade de idosos e pode contribuir para o direcionamento de estratégias de prevenção e tratamento das quedas ${ }^{1,7}$.

Apesar da crescente conscientização acerca do problema que envolve esse medo, são escassas as pesquisas sobre o assunto ${ }^{3,8}$. O principal foco dos estudos sobre as quedas e medo de cair em idosos tem sido nos determinantes físicos1, e pouco se investiga os aspectos psicológicos e sociais envolvidos ${ }^{9}$. Assim, são necessários novos estudos para compreender o efeito de fatores psicossociais no medo de quedas em idosos.

Neri et al. ${ }^{10}$ destacam a relevância do envolvimento social agindo como elemento central do envelhecimento ativo e saudável. Sabe-se que o engajamento do idoso em Atividades Avançadas da Vida Diária (AAVD) possibilita que ele se valorize como indivíduo ativo e produtivo, em função do seu contato com ações e papéis sociais, intelectuais, de lazer, organizacionais e políticos ${ }^{11}$. As AAVD compreendem o conjunto de atividades sociais, produtivas e de lazer, relacionadas com a participação e o envolvimento social, que excedem aquelas de autocuidado, sobrevivência e solução de problemas práticos ${ }^{12}$.

As AAVD estão relacionadas com a diminuição do risco de morte, com o bem-estar emocional, a redução de sintomas depressivos e o melhor funcionamento cognitivo $^{12,13}$. Portanto, tendo em vista estes aspectos, a hipótese básica do presente estudo é que o desempenho de idosos nas AAVD pode também influenciar na diminuição do medo de cair, e, consequentemente, na melhora da sua autoeficácia relacionada às quedas. São escassos os estudos sobre desfechos de natureza psicossocial no âmbito da saúde do idoso, fazendo-se relevante a compreensão dos possíveis fatores associados ao medo de quedas, uma vez que poderá determinar o foco, garantindo a eficácia, de estratégias preventivas.

Embora a relação entre capacidade funcional e medo de quedas em idosos tenha sido investigada, os estudos priorizaram as atividades instrumentais (AIVD) e básicas da vida diária (ABVD) ${ }^{8}$. Dias et al. ${ }^{12}$ apontaram que a avaliação, bem como, estratégias de prevenções e intervenções, das AAVD ainda não ocorre de forma sistemática na clínica gerontológica. Não obstante, os déficits nas AAVD podem ser precursores de perdas em AIVD e ABVD. Nesta direção, cabe investigar a associação do medo de quedas com AAVD entre idosos, para que afirme sobre a relevância de se investir nessas atividades no âmbito da saúde do idoso. Assim, o objetivo deste estudo foi verificar a associação entre medo de quedas e funcionalidade para AAVD em idosos comunitários, controlando os efeitos de fatores sociodemográficos e clínicos.

\section{PROCEDIMENTOS METODOLÓGICOS}

\section{Participantes}

A amostra deste estudo foi composta por indivíduos que participaram da linha de base do Estudo Fragilidade em Idosos Brasileiros - Seção Rio de Janeiro (FIBRA$\mathrm{RJ})^{14}$. A coleta de dados da linha de base foi conduzida de 2009 a 2010, no núcleo de pesquisa da Universidade do Estado do Rio de Janeiro, no município do Rio de Janeiro, RJ, Brasil, com os clientes de uma operadora de saúde. Os critérios de inclusão para os participantes do Estudo FIBRA-RJ eram: ser conveniados a operadora de saúde há pelo menos 12 meses, ter 65 anos ou mais de idade, residir em um dos bairros da Zona Norte da cidade do Rio de Janeiro.

Um total de 9769 indivíduos preencheu esses critérios e, para selecionar a população do estudo, foram estratificados por sexo e faixa etária, sendo a amostra total em cada estrato obtida por amostragem aleatória inversa. O FIBRA-RJ entrevistou 847 idosos em seus próprios domicílios, seguindo o protocolo padrão do estudo (disponível em: www.geronlab.com/). Destes, no presente estudo, foram analisados os dados de 645 idosos, após exclusão dos participantes que preencheram os seguintes critérios: respostas incompletas nas variáveis 
de interesse; ter déficit cognitivo grave sugestivo de demência, evidenciado pelo Mini Exame do Estado Mental ${ }^{15}$; respostas dados por um informante familiar e não pelo próprio idoso; uso de cadeira de rodas ou retenção provisória ou definitiva ao leito; sequelas graves de acidente vascular encefálico, com perda localizada de força e/ou afasia; doença de Parkinson em estágio grave ou instável, com comprometimento grave da motricidade, da fala ou da afetividade; graves déficits de audição ou de visão, que dificultassem fortemente a comunicação; e estar em estágio terminal.

Todos os participantes assinaram o Termo de Consentimento Livre e Esclarecido, que, juntamente com o estudo, foi aprovado pelo Comitê de Ética em Pesquisa do Hospital Universitário Pedro Ernesto, da Universidade do Estado do Rio de Janeiro (processo $n^{\circ}$ 1850-CEP/ HUPE).

\section{Instrumentos}

Medo de quedas. Foi utilizada a FES-I-Brasil, versão do Falls Efficacy Scale - International (FES-I) adaptada e validada para o uso na população brasileira4. A FES-I-Brasil mede a opinião do idoso, sobre o quão preocupado fica, com a possibilidade de cair fazendo cada uma das 16 atividades descritas e como opção de resposta, contem: "nem um pouco", "um pouco preocupado", "muito preocupado" e "extremamente preocupado". Os valores variam de 16 pontos para os indivíduos sem qualquer preocupação em cair a 64 pontos para os indivíduos com preocupação extrema. Esta variável foi dicotomizada de acordo com um corte de 23 pontos.

Desempenho em Atividades Avançadas da Vida Diária (aavd). Foi utilizado um inventário estruturado com uma lista de atividades obtida com base na literatura ${ }^{11,16}$ que busca verificar o envolvimento social dos idosos em 12 atividades de natureza social, de lazer, cultural, organizacional, política e produtiva. Para cada uma das 12 atividades do inventário o participante deveria escolher entre as seguintes opções de resposta: "nunca fez", "parou de fazer" e "ainda faz". Foram considerados com melhor funcionalidade para as AAVD aqueles que responderam "ainda faz" para 7 ou mais atividades. Esta pontuação foi escolhida a partir do valor da mediana da amostra para o total de atividades relatada como "ainda faz".

Variáveis clínicas e funcionais. Número de quedas no último ano $(0,1-2, \geq 3)$; número de comorbidades relatadas como diagnosticadas por um médico, nos últimos 12 meses, dentre as seguintes doenças crônicas: doença do coração, hipertensão arterial sistêmica, acidente vascular cerebral, diabetes mellitus, câncer, artrite ou reumatismo, doenças dos pulmões, depressão e osteoporose (respondiam "sim" ou "não" para cada uma delas), categorizadas em $0-1,2-3$, $\geq 4$; número de medicamentos utilizados regularmente, categorizados em 0-3, 4-6, $\geq 7$; uso de andadores ( $\operatorname{sim} /$ não); auto relato de deficiência visual e auditiva ("se ele/ela tinha dificuldade em ver ou ouvir" [sim/não]). Auto-percepção de saúde foi avaliada com a pergunta, "Em geral, como você diria que sua saúde é?", com as seguintes opções de resposta: muito boa, boa, regular, ruim e muito ruim, reduzidas, para análise, a 2 estratos: muito boa e boa; regular, ruim e muito ruim.

O envolvimento nas atividades básicas de vida diária (ABVD) foi avaliado através da versão brasileira do índice de Katz, em que as respostas apontavam dependência, independência ou necessidade de ajuda para realizar as atividades ${ }^{17}$.

O desempenho em atividades instrumentais de vida diária (AIVD) foi avaliado pela escala de Lawton e Brody ${ }^{18}$, em que se captava dependência ou independência em cada atividade. Os idosos que necessitavam de ajuda para realizarem pelo menos uma das atividades avaliadas foram considerados dependentes. Foi avaliada a síndrome da fragilidade, identificada com os cinco indicadores propostos por Fried et al. ${ }^{19}$ : perda de peso não intencional, baixa força de preensão palmar, sensação de exaustão, baixa velocidade de marcha e baixo nível de atividade física ${ }^{14}$. A partir destes critérios, foram divididos os grupos: nãofrágil, pré-fragil e frágil. Avaliou-se o nível de atividade no último ano (melhor/pior/igual). A partir da versão brasileira reduzida da Escala de Depressão Geriátrica (EDG-15), os idosos tinham as opções de resposta "sim" ou "não" para cada um dos sintomas depressivos descritos. O ponto de corte é de $5 / 6$.

Variáveis sociodemográficas. Gênero, idade, escolaridade. A avaliação foi realizada a partir de questões de autorrelato. Obteve-se a idade a partir da data de nascimento informada pelos idosos, as quais foram agrupadas em quatro faixas: 65-69, 70-74, 75-79, e 80 anos e mais. A informação sobre escolaridade foi derivada da pergunta sobre o total de anos de estudo, depois categorizada em: analfabetos, 1 a 4 anos, 5 a 8 e 9 anos ou mais de escolaridade. Avaliou-se o apoio social pelas questões "Se você precisa ou vai precisar de ajuda para executar qualquer uma dessas atividades da vida diária, você tem a quem recorrer?" e "Você tem um parente, amigo ou vizinho que poderia cuidar de você, se necessário?” (Sim/Não) 


\section{Análise dos dados}

Realizou-se teste de quiquadrado de Pearson para associação entre cada uma das variáveis independentes com medo de quedas. As variáveis associadas significativamente $(\mathrm{p}<0,001)$ com a variável desfecho foram mantidas no modelo de regressão de Poisson. A regressão de Poisson foi utilizada para estimar as razões de prevalência ajustadas (e seus respectivos intervalos de $95 \%$ de confiança), tendo em vista que esta análise poderia oferecer estimativas mais consistentes comparado à regressão logística, dada a elevada prevalência do desfecho analisado. Todos os resultados apresentados de frequências relativas e de medidas de associação foram ponderados pelo peso amostral. Todas as respostas dos participantes foram registradas e analisadas no programa SPSS for Windows 21.0 (Statistical Package for Social Sciences).

\section{RESULTADOS}

Entre os 645 idosos analisados, $263(40,8 \%)$ sentem medo de cair, enquanto $382(59,2 \%)$ não sentem. Na Tabela 1 observam-se as características sociodemográficas associadas com o medo de quedas.

Todas as variáveis socioeconômicas, clínicas e funcionais apresentaram associação significativa com o medo de quedas nas análises bivariadas, inclusive AAVD, cuja associação mostrou que quanto maior a funcionalidade, menor o medo de cair $(\mathrm{RP}=1,05 ; \mathrm{IC}=1,00$ 1,09). No entanto, no modelo multivariado, a direção da associação entre AAVD e o medo de quedas sofreu alteração, ou seja, o que foi observado foi uma maior prevalência do medo de quedas para aqueles com maior funcionalidade em comparação com aqueles com menor funcionalidade para AAVD (Tabela 2).

A prevalência do medo de quedas foi $5 \%$ menor para os idosos com pior funcionalidade para as AAVD comparados aos de maior funcionalidade; $9 \%$ maior para os que são frágeis e 7\% maior para os indivíduos préfrágeis quando comparados com os não frágeis; $8 \%$ maior para os mais velhos (>80 anos) comparados aos de 65-69 anos; $9 \%$ maior para as mulheres comparadas aos homens; $13 \%$ maior para os que caíram 3 vezes ou mais e $14 \%$ maior para os que caíram 1 ou 2 vezes, comparado aos que nunca caíram; $9 \%$ maior para aqueles que percebem a sua saúde como negativa comparados aos que a percebem como positiva; $14 \%$ maior para aqueles com suspeita de depressão em comparação aos que não têm suspeita; $8 \%$ maior para aqueles com 4 ou mais comorbidades comparando com aqueles que têm no máximo 1; e 7\% maior para aqueles que consomem 7 medicamentos ou mais comparados aos que consomem até 3 .

Tabela 1. Características sociodemográficas associadas com o medo de quedas

\begin{tabular}{|c|c|c|}
\hline & \multicolumn{2}{|c|}{ Medo de quedas } \\
\hline & $\begin{array}{l}\text { Não } \\
\text { n (\%) }\end{array}$ & $\underset{\mathbf{n}(\%)}{\operatorname{Sim}}$ \\
\hline \multicolumn{3}{|c|}{$\begin{array}{l}\text { Funcionalidade para } \\
\text { as AAVD* }\end{array}$} \\
\hline Pior & $217(57,2 \%)$ & $169(64,7 \%)$ \\
\hline Melhor & $165(42,8 \%)$ & $94(35,3 \%)$ \\
\hline \multicolumn{3}{|l|}{ Idade* } \\
\hline $65-69$ & $80(20,9 \%)$ & $42(16,0 \%)$ \\
\hline $70-74$ & $103(27,0 \%)$ & $56(21,3 \%)$ \\
\hline $75-79$ & $105(27,4 \%)$ & $58(22,1 \%)$ \\
\hline$\geq 80$ & $94(24,6 \%)$ & $107(40,7 \%)$ \\
\hline \multicolumn{3}{|l|}{ Gênero* } \\
\hline Feminino & $240(58,7 \%)$ & $204(75,1 \%)$ \\
\hline Masculino & $142(41,3 \%)$ & $59(24,9 \%)$ \\
\hline \multicolumn{3}{|l|}{ Renda* } \\
\hline$\leq 1$ & $45(11,8 \%)$ & $37(14,1 \%)$ \\
\hline $1,01-3$ & $57(14,9 \%)$ & $38(14,2 \%)$ \\
\hline $3,01-5$ & $103(27,0 \%)$ & $62(23,8 \%)$ \\
\hline $5,01-10$ & $107(28,0 \%)$ & $88(33,4 \%)$ \\
\hline$>10$ & $70(18,3 \%)$ & $38(14,5 \%)$ \\
\hline \multicolumn{3}{|c|}{ Escolaridade* } \\
\hline Analfabeto & $5(1,0 \%)$ & $9(3,0 \%)$ \\
\hline $1-4$ & $57(14,8 \%)$ & $45(17,3 \%)$ \\
\hline $5-8$ & $70(18,2 \%)$ & $65(24,6 \%)$ \\
\hline$\geq 9$ anos & $250(66,0 \%)$ & $144(55,1 \%)$ \\
\hline \multicolumn{3}{|c|}{ Histórico de quedas* } \\
\hline 0 & $311(81,2 \%)$ & $157(59,7 \%)$ \\
\hline $1-2$ & $63(16,7 \%)$ & $86(32,7 \%)$ \\
\hline$\geq 3$ & $8(2,1 \%)$ & $20(7,6 \%)$ \\
\hline
\end{tabular}


Tabela 2. Razão de prevalência e intervalos de $95 \%$ de confiança para a associação entre funcionalidade para AAVD, medo de quedas, características clínicas e psicossociais

\begin{tabular}{|c|c|c|}
\hline & RP (IC 95\%) & p-valor \\
\hline \multicolumn{3}{|l|}{$\begin{array}{l}\text { Funcionalidade para as } \\
\text { AAVD }\end{array}$} \\
\hline Pior & $0,95(0,91-0,99)$ & $<0,05$ \\
\hline Melhor & 1 & \\
\hline \multicolumn{3}{|l|}{$\begin{array}{l}\text { Escala de Fragilidade de } \\
\text { Fried }\end{array}$} \\
\hline Frágil & $1,09(1,02-1,17)$ & $<0,05$ \\
\hline Pré-frágil & $1,07(1,03-1,12)$ & $<0,05$ \\
\hline Não caso & 1 & \\
\hline \multicolumn{3}{|l|}{ Idade } \\
\hline$\geq 80$ & $1,08(1,02-1,15)$ & $<0,05$ \\
\hline $75-79$ & $0,97(0,91-1,03)$ & 0,255 \\
\hline $70-74$ & $0,96(0,90-1,03)$ & 0,217 \\
\hline $65-69$ & 1 & \\
\hline \multicolumn{3}{|l|}{ Gênero } \\
\hline Feminino & $1,09(1,04-1,14)$ & $<0,05$ \\
\hline Masculino & 1 & \\
\hline \multicolumn{3}{|l|}{ Histórico de quedas } \\
\hline$\geq 3$ & $1,13(1,03-1,24)$ & $<0,05$ \\
\hline $1-2$ & $1,14(1,09-1,19)$ & $<0,05$ \\
\hline 0 & 1 & \\
\hline \multicolumn{3}{|l|}{ Autopercepção de saúde } \\
\hline Negativa & $1,09(1,05-1,14)$ & $<0,05$ \\
\hline Positiva & 1 & \\
\hline \multicolumn{3}{|l|}{ EDG } \\
\hline Com suspeita de depressão & $1,14(1,09-1,19)$ & $<0,05$ \\
\hline Sem suspeita de depressão & 1 & \\
\hline \multicolumn{3}{|l|}{ Número de comorbidades } \\
\hline$\geq 4$ & $1,08(1,01-1,17)$ & $<0,05$ \\
\hline $2-3$ & $1,03(0,98-1,08)$ & 0,253 \\
\hline $0-1$ & 1 & \\
\hline \multicolumn{3}{|l|}{ Número de medicamentos } \\
\hline$\geq 7$ & $1,07(1,01-1,13)$ & $<0,05$ \\
\hline $4-6$ & $1,02(0,97-1,07)$ & 0,502 \\
\hline $0-3$ & 1 & \\
\hline
\end{tabular}

RP (IC 95\%): Razão de prevalência e intervalo de confiança em nível de $95 \%$ obtido com regressão de Poisson e ponderado pelo peso amostral, ajustado por AAVD, ABVD, AIVD, idade, escolaridade, fragilidade, gênero, histórico de quedas, apoio social, autopercepção de saúde, classificação na EDG-15, número de comorbidades, número de medicamentos, dependência de andadores, deficiência visual e auditiva.

\section{DISCUSSÃO}

Baseado nas evidências de que o engajamento em AAVD traz diversos benefícios aos idosos ${ }^{12,13}$, esperava-se a associação entre a maior funcionalidade para as AAVD com a menor prevalência do medo de quedas. Contudo, diferente do esperado, os resultados do presente estudo mostraram que o medo de cair entre os idosos com pior funcionalidade para AAVD foi menor que entre os idosos com melhor funcionalidade para AAVD, isto é, o grupo menos engajado apresentou maior autoeficácia relacionada às quedas.

A associação entre o menor medo de cair e pior AAVD, pode ser devido a menor exposição dos idosos com pior funcionalidade aos perigos e adversidades ambientais vivenciadas ao sair de casa. Além disso, a associação obtida pode corresponder a um mecanismo adaptativo destes idosos para lidarem com prejuízos associadas ao aumento da idade, conforme formulado por Baltes e Baltes $^{20}$ na teoria lifespan, sobre as estratégias de seleção, otimização e compensação (teoria SOC). Segundo estes autores, a estratégia da seleção envolve dirigir-se a metas hierarquizadas a partir da compatibilidade com os recursos disponíveis, a otimização significa a utilização dos recursos internos e externos que se dispõe para o alcance das metas selecionadas e a compensação envolve o uso de mecanismos e alternativas alcançáveis para manutenção do funcionamento. Com base neste mecanismo, pode-se supor que indivíduos mais velhos podem selecionar as AAVD para as quais são mais competentes e que exigem menos de seus recursos físicos, emocionais e sociais. Assim, a diminuição no nível de atividades seria um processo seletivo benéfico no sentido de que os recursos do idoso poderiam ser reservados para as funções associadas a maior bem-estar; promovendo minimização de perdas e a maximização dos ganhos.

Acrescenta-se à discussão, as explicações da teoria da seletividade sócio-emocional, derivada da teoria SOC, para o fato de que os idosos, em função da mudança na sua perspectiva de tempo futuro, redistribuem seus próprios recursos socioemocionais ${ }^{21}$. Segundo esta teoria, a amplitude das redes sociais é reduzida ativamente pelos idosos e o contato social ocorre, preferencialmente, com as pessoas que oferecem significativas experiências emocionais positivas, o que leva à otimização do bemestar psicológico na velhice. Os pressupostos desta teoria podem explicar a diminuição do nível de AAVD observada no presente estudo, de forma que motivados por uma regulação emocional, os idosos podem privilegiar atividades em seus próprios domicílios, abandonando as 
atividades que não são com o grupo social mais próximo. Nesse sentido, faz-se relevante os achados da presente pesquisa para compreensão de fenômenos psicológicos, como autoeficácia relacioanada a eventos comuns na velhice, como as quedas. Além disso, compreender esses fenômenos com base em modelos da psicologia do envelhecimento pode aprimorar as intervenções gerontológicas, uma vez que estas teorias auxiliam na discussão a respeito das mudanças que ocorrem com o aumento da idade.

É sabido que não só o estado físico e as habilidades atuam na autoeficácia, como também a influência social, as experiências prévias e a observação da experiência de terceiros5. Sendo assim, é possível supor que os idosos com pior funcionalidade para as AAVD estão sendo privados de experiências que aumentam as chances de ter medo de quedas. Pode-se supor ainda que estes estão se esquivando dessas atividades, uma vez que a autoeficácia também pode funcionar como preditora de comportamentos futuros.

É importante se atentar para a atual "glamourização dos grupos de convivência para a terceira idade" e a "socialização forçada" que obrigam os idosos a participarem de atividades socializadoras, desconsiderando a heterogeneidade presente na velhice ${ }^{22}$. Não obstante tenham sido evidenciados os ganhos do engajamento social para a saúde física e mental de idosos, a depender do perfil funcional do idoso, pode ser recomendável a redução de algumas atividades, entendendo a diminuição do envolvimento em AAVD como uma estratégia preventiva e de promoção do bem-estar. Consoante ao exposto, Pereira et al. ${ }^{23}$ descreveram que, na ocorrência de quedas, a percepção do idoso a respeito de locais públicos inseguros se torna um fator protetor. Assim, faz-se necessário o investimento em tornar o espaço urbano mais acessível e seguro para a população mais velha, uma vez que idosos em contato com espaços externos ao ambiente doméstico podem percebê-los como espaços inseguros, gerando o aumento do medo de cair.

$\mathrm{Na}$ presente pesquisa, os idosos com pior funcionalidade para as AAVD apresentaram menor prevalência de quedas que aqueles com melhor funcionalidade para as AAVD. Entende-se que, no ambiente externo, é reduzido o senso de controle do idoso sobre o evento que considera inseguro, o que pode estar associado a consequências mais graves em caso de quedas e, consequentemente, impactando no medo de quedas. $\mathrm{O}$ aumento do temor de quedas está mais relacionado ao agravo causado pela queda do que com a queda propriamente dita. No presente estudo, em decorrente da insuficiência da amostra de idosos que caíram, as consequências das quedas não foram estudadas. Assim, sugere-se que futuros estudos avaliem as consequências das quedas como uma variável interveniente na relação entre autoeficácia relacionada às quedas e as AAVD.

É importante destacar, como limitação do estudo, que algumas das AAVD do questionário da pesquisa podem ser pouco realizadas no contexto sociocultural da população de estudo. As atividades de participação em grupos para terceira idade, por exemplo, teve baixa frequência na população de estudo; $62,6 \%$ dos idosos nunca a fizeram. Segundo Pinto e Neri ${ }^{24}$, o envolvimento nesse tipo de programa de extensão requer disponibilidade desses recursos, bem como compatibilidade com ocupações anteriores; com níveis de escolaridade e renda; com os compromissos familiares; e com hábitos e interesses de homens e mulheres idosos. A frequência de participação também foi baixa em trabalhos voluntários (64,2\% dos idosos nunca o realizaram), podendo também ser explicado a partir do exposto por Pinto e $\mathrm{Neri}^{24}$ de que o voluntariado ainda não é disseminado na cultura brasileira.

Em um estudo de revisão, Dias et al. ${ }^{12}$ destacaram a dificuldade na criação de uma escala universal para medir a funcionalidade nas AAVD, em função da alta variabilidade entre os indivíduos. As autoras ainda apontam que a escolha por esse tipo de atividade no cotidiano é influenciada por fatores subjetivos, culturais e psicossociais, estilo de vida, condições e motivação para a escolha. Ademais, consoante à ponderação de Oliveira et al. ${ }^{16}$, a frequência/intensidade de envolvimento com essas atividades poderia ser uma medida mais fidedigna de avaliação da funcionalidade que a quantidade de AAVD realizadas. No presente estudo, foi utilizado um inventário que vem sendo amplamente utilizado no contexto brasileiro $^{13,16}$ para descrever o engajamento de idosos em atividades sociais, mas recomenda-se o investimento de pesquisadores na construção de instrumentos capazes de avaliar de forma mais sistemática as AAVD.

A população de estudo foi composta de idosos saudáveis e não houve comparação com idosos que apresentam maiores níveis de perdas funcionais nas atividades básicas e instrumentais de vida diária. Futuros estudos devem ser conduzidos para avaliar a associação da autoeficácia relacionada às quedas em idosos com uma maior variabilidade de perfis funcionais. Antes e colaboradores8, por exemplo, observaram que com um perfil de maior comprometimento da saúde física, a redução de contatos sociais pode estar associada ao maior medo de quedas.

No que se refere às características clínicas e psicossociais, os resultados do presente estudo corroboram 
achados que apontaram o aumento da idade, o sexo feminino, o histórico de quedas, a suspeita de depressão, a pior percepção de saúde e o pior nível de atividade como fatores associados ao aumento do medo de quedas ${ }^{1,3,8}$. Cabe destacar que em estudo anterior, com a mesma amostra da presente investigação, Malini et al. ${ }^{25}$ já haviam apontado estas associações entre diversas variáveis clínicas, funcionais e psicológicas com o medo de quedas. Contudo, o presente estudo mostrou que, sob efeito das AAVD, algumas destas características demográficas e clínicas perdem seu efeito, como: a escolaridade, a dependência funcional para atividades básicas e instrumentais, a deficiência auditiva e visual, o uso de bengala e ter com quem contar. Pode-se concluir, então, que é de extrema relevância o impacto da funcionalidade para as AAVD sobre o medo de quedas, de modo que esses achados contribuem para ampliar a compreensão da saúde do idoso, contemplando o estudo de fenômenos não apenas de natureza física, como também psicológica. Nessa direção, o modelo proposto extrapola as explicações dos modelos biomédicos, ao buscar explicações para questões gerontológicas com a inclusão de determinantes biopsicossociais, ou seja, vai além das dimensões biológicas, as quais são mais estudadas no âmbito da saúde do idoso.

Entre os pontos fortes do presente estudo, destacase ser uma amostra comunitária com grande número de indivíduos e ter investigado a associação entre AAVD com a FES-I-Brasil, tema ainda inexplorado na área gerontológica. O efeito das AAVD sobre o medo de quedas foi contrário ao que se formulou como hipótese inicial nessa investigação e demonstra a complexidade da natureza das AAVD no âmbito da saúde do idoso. Estudos dentro das temáticas abordadas se justificam a medida que estratégias de prevenção e promoção de saúde não devam se resumir ao simples incentivo destas atividades entre idosos. Sugere-se que mais investigações sejam conduzidas para ampliar a compreensão dos efeitos deste engajamento na velhice, bem como contribuir para o avanço da psicologia do envelhecimento.

Agradecimento: Conselho Nacional de Pesquisa - Brasil (CNPq - 555087/2006-9), Fundação Carlos Chagas Filho para Pesquisa Suporte do Estado do Rio de Janeiro, Brasil (FAPERJ - E- 26/171.469/2006, E-26/110.294/2007), Fundação de Amparo à Pesquisa do Estado de Minas Gerais - FAPEMIG (CHE - APQ-01145-14).

Agências de fomento: Conselho Nacional de Pesquisa - CNPq ( No 555087/2006-9) e Fundação de Amparo à Pesquisa do Estado de Minas Gerais - FAPEMIG (CHE - APQ-01145-14).

Contribuições de cada autor na elaboração do artigo: JF Souto - Elaboração do manuscrito; realizou a análise estatística, interpretação dos dados e redigiu o manuscrito. PCC Ribeiro - Participou da elaboração do projeto de pesquisa; redigiu o manuscrito; supervisionou a análise de dados; e finalizou o texto do manuscrito. RA Lourenço - Participou da elaboração do projeto de pesquisa e finalizou a revisão crítica do manuscrito.

\section{REFERÊNCIAS}

1. Scheffer AC, Schuurmans MJ, Dijk N, Hooft TD, Rooij SE. Fear of falling: measurement strategy, prevalence, risk factors and consequences among older persons. Age Ageing. 2008;37(1):19-24. doi: 10.1093/ageing/afm169.

2. Dias RC, Freire MTF, Santos EGS, Vieira RA, Dias JMD, Perracini MR. Características associadas à restrição de atividades por medo de cair em idosos comunitários. Braz J Phys Ther. 2011;5(15):406-13. doi: 10.1590/S141335552011000500011 .

3. Malini FM, Lopes CS, Lourenço RA. Medo de quedas em idosos: uma revisão da literatura. Pedro Ernesto University Hosp J. 2014;13(2):38-44. doi: 10.12957/rhupe.2014.10127.

4. Camargos FFO, Dias RC, Dias JMD, Freire MTF. Adaptação transcultural e avaliação das propriedades psicométricas da Falls Efficacy Scale - International em idosos brasileiros (FESI-BRASIL). Braz J Phys Ther. 2010;3(14):237-43. doi: 10.1590/ S1413-35552010000300010.
5. Bandura A, Adams NE, Beyer J. Cognitive processes mediating behavioral change. J Personality Social Psychol. 1977;35(3):125-39. doi: 10.1037/0022-3514.35.3.125.

6. Tinetti ME, Richman D, Powell L. Falls efficacy as a measure of fear of falling. J Gerontol. 1990;45(1):239-43. doi: 10.1093/ geronj/45.6.P239.

7. Delbaere K, Close JC, Mikolaizak AS, Sachdev PS, Brodaty H, Lord SR. The Falls Efficacy Scale International (FES-I): a comprehensive longitudinal validation study. Age Ageing. 2010;39(2):210-6. doi: 10.1093/ageing/afp225.

8. Antes DL, Schneider IJC, Benedetti TRB, D'Orsi E. Medo de queda recorrente e fatores associados em idosos de Florianópolis. Cad Saúde Pública. 2013;29(4):758-68. doi: 10.1590/S0102-311X2013000400013.

9. Hadjistavropoulos T, Delbaere K, Fitzgerald TD. Reconceptualizing the role of fear of falling and balance confidence in fall risk. J Aging Health. 2011;23(1):3-23. doi: 


\section{$10.1177 / 0898264310378039$.}

10. Neri AL, Costa TB, Marincolo JCS, Ribeiro LHM. Atividade física, envolvimento social, produtividade e satisfação com a vida. In: Neri AL, Guariento ME. Fragilidade, saúde e bemestar em idosos: dados do estudo FIBRA Campinas. Campinas: Alínea; 2011.

11. Reuben D, Laliberte L, Hiris J, Mor V. A hierarchical exercise scales to measure function at the Advanced Activities of Daily Living (AADL) level. J Am Geriatr Soc. 1990;38(8):855-61. doi: 10.1111/j.1532-5415.1990.tb05699.x.

12. Dias EG, Duarte Y, Almeida MHM, Lebrão ML. Caracterização das atividades avançadas. Rev Ter Ocup Univ São Paulo. 2011;1(22):45-51. doi: 10.11606/issn.2238-6149.v22i1p45-51.

13. Sposito G, Neri AL, Yassuda MS. Atividades avançadas de vida diária (AAVDs) e o desempenho cognitivo em idosos residentes na comunidade: dados do estudo FIBRA Polo UNICAMP. Braz J Ger Gerontol. 2016;19(1):7-20. doi: 10.1590/1809-9823.2016.15044.

14. Moreira VG, Lourenço RA. Prevalence and factors associated with frailty in an older population from the city of Rio de Janeiro, Brazil: the FIBRA-RJ Study. Clinics. 2013;68(7):97985. doi: 10.6061/clinics/2013(07)15.

15. Brucki SMD, Nitrini R, Caramelli P, Bertolucci, PHF, Okamoto IH. Sugestões para o uso do Mini-exame do Estado Mental no Brasil. Arq Neuro-Psiquiatr. 2003;61(3-B):777-81. doi: 10.1590/S0004-282X2003000500014.

16. Oliveira EM, Silva HS, Lopes A, Cachioni M, Falcão DVS, Batistone SST, et al. Atividades avançadas de vida diária (aavd) e desempenho cognitivo entre idosos. Psico-USF. 2015;1(20):109-20. doi: 10.1590/1413-82712015200110.

17. Lino VTS, Pereira SRM, Camacho LAB, Ribeiro Filho ST, Buksman S. Adaptação transcultural da escala de independência em atividades de vida diária (escala de Katz). Cad Saúde Pública. 2008;24(1):103-12. doi: 10.1590/S0102-

\section{X2008000100010}

18. Lawton MP, Brody EM. Assessment of older people: selfmaintaining and instrumental activities of daily living. Gerontologist. 1969;9(3):179-86. doi: 10.1093/geront/9.3_ Part_1.179.

19. Fried LP, Tangen CM, Walston J, Newman AB, Hirsch C, Gottdiener J, et al. Frailty in older adults: evidence for a phenotype. J Gerontol A Biol Sci Med Sci. 2001;56(3):146-56. doi: 10.1093/gerona/56.3.M146.

20. Baltes PB, Baltes MM. Psychological perspectives on successful aging: The model of selective optimization with compensation. In: Baltes PB, Baltes MM. Successful aging. Perspectives from behavioral sciences. Cambridge: Cambridge University Press; 1990. p.1-34.

21. English T, Carstensen LL. Selective narrowing of social networks across adulthood is associated with improved emotional experience in daily life. Int J Behav Dev. 2014;38(2):195-202. doi: 10.1177/0165025413515404.

22. Moura GA, Souza LK. Autoimagem, socialização, tempo livre e lazer: quatro desafios à velhice. Textos Contextos (Porto Alegre). 2012;11(1):172-83. doi: 10.15448/1677-9509.

23. Pereira GN, Morsch P, Lopes DG, Trevisan MD, Ribeiro A, Navarro JH, et al. Social and environmental factors associated with the occurrence of falls in the elderly. Cien Saúde Coletiva. 2013;18:3507-14. doi: 10.1590/S1413-81232013001200007.

24. Pinto JM, Neri AL. Doenças crônicas, capacidade funcional, envolvimento social e satisfação em idosos comunitários: Estudo Fibra. Cien Saúde Coletiva. 2013;18(12):3449-60. doi: 10.1590/S1413-81232013001200002.

25. Malini FM, Lourenço RA, Lopes CS. Prevalence of fear of falling in older adults, and its associations with clinical, functional and psychosocial factors: The Frailty in Brazilian Older People-Rio de Janeiro Study. Ger Gerontol Int. 2015;16(3):336-44. dói: 10.1111.ggi.12477. 